Peter Brockmeier/Carola Veit (Hrsg.) $\cdot$ Komik und Solipsismus im Werk Samuel Becketts 


\author{
Peter Brockmeier \\ Carola Veit (Hrsg.)
}

\title{
Komik und Solipsismus im Werk Samuel Becketts
}


Die Deutsche Bibliothek - CIP-Einheitsaufnahme

Komik und Solipsismus im Werk Samuel Becketts/

Peter Brockmeier; Carola Veit (Hrsg.)

- Stuttgart: M und P, Verl. für Wiss. und Forschung, 1996

ISBN 978-3-476-45174-3

ISBN 978-3-476-45174-3

ISBN 978-3-476-04269-9 (eBook)

DOI $10.1007 / 978-3-476-04269-9$

Dieses Werk ist einschließlich aller seiner Teile geschützt. Jede Verwertung außerhalb der engen Grenzen des Urheberrechtsgesetzes ist ohne Zustimmung des Verlages unzulässig und strafbar. Das gilt insbesondere für Vervielfältigungen, Übersetzungen, Mikroverfilmungen und Einspeicherung in elektronischen Systemen.

M \& P Verlag für Wissenschaft und Forschung ein Verlag der J.B. Metzlerschen Verlagsbuchhandlung und Carl Ernst Poeschel Verlag GmbH in Stuttgart

(C) 1997 Springer-Verlag GmbH Deutschland

Ursprünglich erschienen bei J.B. Metzlersche Verlagsbuchhandlung und Carl Erust Poeschel

Verlag $\mathrm{GmbH}$ in Stuttgart 1997 


\section{In uitam bumedrasn.}

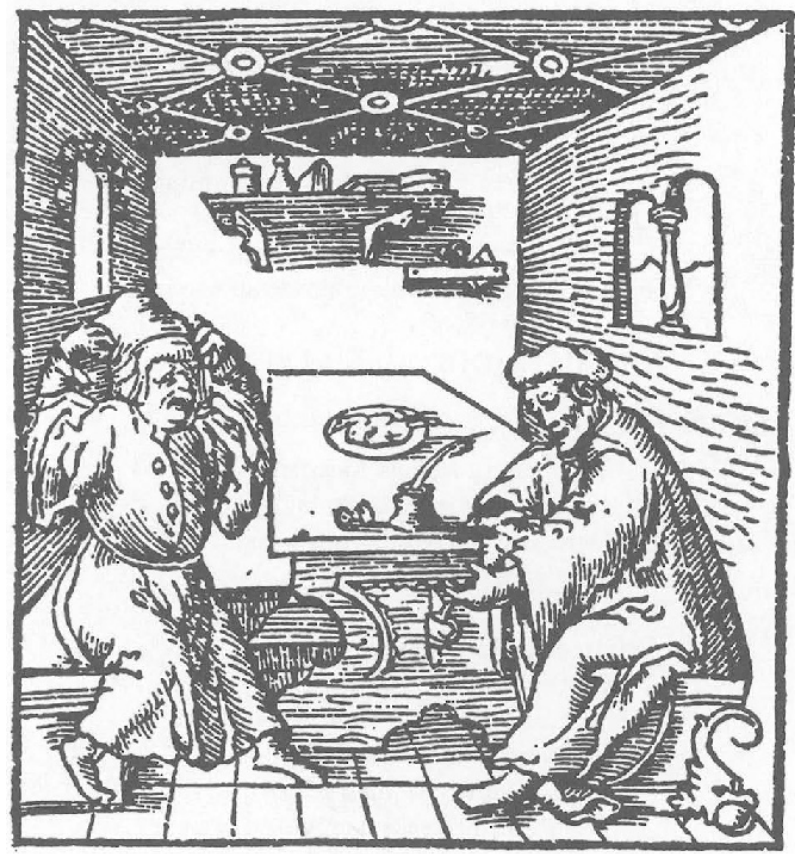

Plus folit c humane nume defle incommoda wite, Heraclice, faxtet pluribus illa malis. Tu rurjus, , quando alids, extolle vachinrum Democrite, illa magns ludicra fáta fuit. Intered hec cernens seditur, quu denisj; trount Fine fledin, ahit tean quomodó pplene ioar, 


\section{Democrit und Heraclit \\ Optimismus und Pessimismus}

\section{IN VITAM HUMANAM.}

Plus solito humanae nunc defle incomoda vitae

Heraclite, scatet pluribus illa malis.

Tu rursus, si quando alias extolle cachinnum,

Democrite, illa magis ludicra facta fuit.

Interea haec cernens meditor, qua denique tecum.

Fine fleam, aut tecum quomodo splene iocer.

\section{VOM MENSCHLICHEN LEBEN.}

Heraclite du alter greiß

Thu jetzt beweinen mit mehr fleiß

Deß Menschlichen Lebens vnglück

Dann es steckt voller böser tück

$\mathrm{Du}$ aber widerumb erschell

Democrite dein glechter hell

Dann lecherlicher zu keiner zeit

Gewesen ist als jetzt die geit

Dieweil ich aber dieses sich

Betracht ich bey mir fleissiglich

$\mathrm{Ob}$ ichs mit dir beweinen sol

Oder mit dir verlachen wol.

(umseitige Abb. aus: A. Alciatus, Emblematum Libellus, Einl. v. A. Buck, Darmstadt 1991, S. 212;

Texte aus: Emblemata. Handbuch zur Sinnbildkunst des XVI. und XVII. Jabrbunderts, hg. v. A. Henkel u. A. Schöne, Stuttgart 1978) 


\section{INHALTSVERZEICHNIS}

Vorwort

Raymond Federman

Samuel Beckett ou le Bonheur en enfer

Carola Veit

Das Absurde in Becketts Werk - Reconsidered

Martin Brunkhorst

Scherz, Satire, Ironie und tiefere Bedeutung in

Krapp's Last Tape

Manfred Schmeling

Humor ohne Versöhnung - Körpersprache in Becketts

Erzählwerk

Wilhelm Füger

Laughter is not quite the word. Arten und Funktionen

des Lachens in Becketts Frühwerk

Konrad Schoell

en face le pire jusqu'à ce qu'il fasse rire. Komik und Humor bei Beckett

Chris Rauseo

Das umgekehrte Erhabene und die Umkehrung seiner sprachlichen Gestalt. Zu Becketts zweisprachigem Humor 
Erika Tophoven

Samuel Becketts Wanderjahre in Deutschland (1928-1937)

\section{Robert Kudielka}

An art unresentful of its insuperable indigence... Das Paradigma der modernen Malerei in Becketts Poetik

Therese Fischer-Seidel

Selbstreflexion und Wahrnehmung in Samuel Becketts

More Pricks than Kicks und Catastrophe

Marianne Kesting

Solipsismus und Ich-Vervielfältigung. Aspekte der literarischen

Figur bei Beckett

Jean Bessière

Les figures réversibles du solipsisme dans Le Dépeupleur,

Compagnie, Mal vu mal dit, Cap au pire, Soubresauts

Peter Brockmeier

Komisches Unglück: Erzähler und Erzählfiguren

Samuel Becketts 


\section{VORWORT}

Am 7. und 8. Juni dieses Jahres fand an der Humboldt-Universität zu Berlin unter dem Titel Solipsismus und Humor. Zur Erinnerung an Samuel Becketts 90. Geburtstag ein Kolloquium statt, dessen Beiträge wir hier veröffentlichen. Damit haben wir - nach dem Tod des Dichters im Jahr 1989 die Anregung des Kolloquiums aufgegriffen, das im April 1986 in Kassel zu seinem 80. Geburtstag stattgefunden hat und das 1988 von Martin Brunkhorst, Gerd Rohmann und Konrad Schoell unter dem Titel Beckett und die Literatur der Gegenwart veröffentlicht worden ist. Neben Konrad Schoell und Martin Brunkhorst haben auch Marianne Kesting und Manfred Schmeling an beiden Kolloquien teilgenommen. Samuel Beckett kannte beide Städte seit seinen "Wanderjahren in Deutschland". Durch die Ortswahl und durch den Beitrag von Erika Tophoven, in dem Leseerfahrungen des Dichters skizziert werden, möchten wir daran erinnern, daß Beckett seinem Werk auch die deutsche Literatur und Kultur eingefügt hat. Wir möchten mit dieser Veröffentlichung vor allem das anhaltende Interesse deutsch-, englisch- und französischsprachiger Übersetzer, Anglisten, Romanisten, Komparatisten oder Kunstwissenschaftler für ein sparsam gestaltetes, aber fast unausschöpflich sinnreiches Werk fortsetzen.

Blättert man die Titel der Untersuchungen durch, die in dem Band Critique of Beckett Criticism: A Guide to Research in English, French and German ${ }^{1}$ zusammengestellt worden sind, so entdeckt man das eine Problem unseres Themas: den Solipsismus oder das andere: die Komik jeweils unter einer Reihe verschiedener Begriffe, die aber die gleiche Sache oder Fragestellung suggerieren: "The Language of the Self"; "A Cartesian Novel"; "Tendenz zum Monologischen"; "Microcosm of the Mind"; "Rückbezüglichkeit"; "The Solipsistic Novel"; "Temptation of Solipsism";

1 Hg. v. P.J. Murphy, W. Huber, R. Breuer, K. Schoell, Columbia S.C., 1994, S. $122-$ 162. 
"Self and Language"; "Subjektivität als Selbsterfindung"; "Reflections in the Mind's Eye"; "Une voix qui s'écoute se taire". Für die Aspekte des Komischen, Grotesken, Parodistischen, Ironischen sind etwa Studien mit den folgenden Titeln zuständig: "The Comic Gamut"; "Farce métaphysique"; "Kunst der Paradoxie"; "The Shape of Paradox"; "Grotesque et signification"; "Ironie et parodie"; "Dialectical Laughter"; "Spiel und Spiele"; "Know Happiness: Irony in Ill Seen Ill Said"; "absurde Farce"; "The Humour of Samuel Beckett"; "The Comedy of Entropy"; "The Tragic Comedians"; "fabulazione, comicità e scrittura". Diese Titel lassen sich wiederum in einen deutlichen Kontrast zu Wahrnehmungsweisen einer "école misérabiliste" (Raymond Federman) bringen: "Writing in the Shit"; "Retour du tragique"; "Structure hystérique et obsessionnelle"; "Unhappy Consciousness"; "Samuel Beckett and the Pessimistic Tradition"; "Einübung ins Nichts"; "Entfremdung".

Gewiß steht die Entfremdung in einem ursächlichen Zusammenhang mit der "schizoiden Selbstrecherche" (Marianne Kesting). Wenn wir aber die Studien von Ruby Cohn, Frederick J. Hoffmann und Marianne Kesting selbst aufschlagen, die beiden Fragestellungen unseres Titels schon 1962 ihre Aufmerksamkeit gewidmet haben, so werden wir feststellen, daß auch sie in erster Linie die ästhetische Konstruktion oder die literarische Besonderheit der Werke selbst untersucht haben. Sie wollten weder mit der einen noch mit der anderen Fragestellung einer naiv naturalistischen Lektüre oder Rezeption der Texte das Wort reden - so als hätte Becketts Schilderung des Elends etwas mit Zola, als hätten seine Ironie und sein "humour" allzuviel mit Feydeau zu tun! Eine solche Reduktion der Probleme wollen wir nicht bewirken, wenn wir scheinbar Widersprüchliches mit einem "und" verbinden: die Komik der Texte mit der Selbst-Darstellung - oder der Selbstbehauptung, welche Arthur Koestler wiederum als notwendigen Impuls des Komischen aufgefaßt hat. Wir möchten als erstes daran erinnern, daß Beckett Selbstdarstellung und Selbstreflexion als freies Spiel der künstlerischen Phantasie verstanden hat. 
Der "Geist" des Titelhelden Murphy - über die Ironie und den Humor dieses Romans scheint man sich einig zu sein - zeichnet sich nach den Worten des Erzählers durch die "absolute Freiheit", das "Vergnügen der Kontemplation" und das aggressive Spiel mit der "physischen Erfahrung" aus: "Ici le fiasco physique tout entier se transformait en succès fou." Entscheidungen zwischen Wahr oder Falsch, Gut oder Böse, Schön oder Häßlich erscheinen also hinfällig. In ähnlichem Sinn hat Frederick J. Hoffmann Becketts Erzählen vom inneren Selbst beschrieben und als eine Form des Erzählens verstanden, die sich geschichtlich von "der Notwendigkeit [...] der metaphysischen Spekulation" befreit hat. ${ }^{2}$ Der Zusammenhang mit Becketts Poetik erscheint noch plausibler, wenn wir formulieren: Sein Erzählen folgt nicht dem "Postulat einer auf Sinneinheit gerichteten endlichen Vernunft". 3

Daß die Verbindung zwischen Komik und Solipsismus nur scheinbar widersprüchlich ist, hat auch Ruby Cohn in ihrer wegweisenden Studie beobachtet: "But Beckett's laughter - the laughter he expresses and the laughter he evokes - is a mask for, not a release from despair." ${ }^{4}$ Wenn sie

2 "The point is that, without the necessity of God, or of the metaphysical speculation consequent upon His discernible presence within a society of selves, the novel becomes, first, a novel of manners - that is of understood conventions and an understanding imagination; next, a novel of conscious selves maneuvering through spaces occupied by objects that are either testimonies of or challenges to them; and, finally, of selves almost exclusively, of the 'interior selves' of interior monologue. This is a steady development away from metaphysics and toward self-definition sub specie $d u$ rationis." (F.J. Hoffmann, Samuel Beckett: The Language of the Self, Carbondale, Ill. 1962, S. 67f.) M. Kesting hat 1962 diese neue Form des Selbst-Erzählens als "ein endloses Spiel" mit dem Wechsel der Identitäten" und u.a. mit der Aussage vorgestellt: "Raum und Zeit durchdringen den Roman frei [...] sie erscheinen im Spiegel des 'inneren Bewußtseins', der Erinnerung oder des berühmten 'monologue intérieur', in dauerndem Perspektivwechsel." (M. Kesting, "Das Romanwerk Samuel Becketts", zitiert nach: dies., Vermessung des Labyrinths. Studien zur modernen Ästhetik, Frankfurt a.M. 1965, S. 70, 68)

3 M. Forschner, in: Lexikon der Ethik, hg. v. O. Höffe u.a., München ${ }^{4} 1992$, s.v. "Gott", S. 105.

4 R. Cohn, Samuel Beckett: The Comic Gamut, New Brunswick, N.J. 1962, S. 287. 
darüber hinaus und auch zurecht festgestellt hat, daß Formlosigkeit und Zusammenhanglosigkeit der sogenannten Trilogie Becketts - Molloy, Malone meurt, L'Innommable - ein Hohn auf den gut gemachten Roman sei$\mathrm{en}^{5}$, so haben wir das als Anregung aufgefaßt, um unsere Frage zu stellen: Besteht das Kompositionsprinzip Beckettscher Texte darin, daß die Selbstreflexion als notwendigerweise komische Selbstbehauptung dargestellt wird? Wir sind selbstverständlich nicht der Meinung, daß eine solche Frage mit Ja oder Nein beantwortet werden kann. Aber die Autoren unseres Bandes haben eine Fülle an Material und eindringliche Überlegungen vorgetragen, so daß man alle Texte Becketts nach diesem oder einem entsprechenden Modell, in jedem Fall aber grundsätzlicher als bisher nach dem Prinzip seiner Schreibweise wird befragen können.

In den folgenden Beiträgen wird das Problem des Zusammenhangs zwischen der Selbstdarstellung und der Komik unterschiedlich gewichtet. Entweder stehen Konzepte des Komischen, literarische Verfahren ihrer Anwendung und die Wirkung auf Leser und Zuschauer im Mittelpunkt; oder es werden verschiedene Texte unter produktionsästhetischen und narratologischen Gesichtspunkten analysiert. Eine Überleitung zwischen diesen beiden Orientierungen hat Erika Tophoven hergestellt, indem sie nicht allein auf die Fähigkeit Becketts aufmerksam macht, polyglott zu kombinieren und Texte zu produzieren, sondern indem sie durch ein längeres Zitat aus einem unveröffentlichten "Notebook" dem künstlerischen Schaffen eine poetologische Absicht zugeordnet hat. Diese ließe sich etwa mit dem von Beckett abgeschriebenen Satz Fritz Mauthners begründen: "Ein Sichversenken in die bloße Stimmung ist wohl eine Weile möglich; dann aber sucht der Grübler immer wieder wie ein Lyriker doch die Stimmung in einem armen Worte festzuhalten und muß ins Leere greifen, wenn er nicht mehr an das Wort glaubt."

5 Ebd., S. 288: "Amorphous and non-concatenated, the volumes of the French trilogy mock the well-made novel. Pointed and repetitive, Comment c'est mocks the traditional novel by diminishing plot to human situation." 
Raymond Federman plädiert in seinem einführenden Essay zwar dafür, daß eine "miserabilistische" Betrachtungsweise des Beckettschen Werkes unangemessen sei; aber seine Vergegenwärtigung des Komischen - die er der Forschung seit Ruby Cohn zuordnet - konfrontiert uns mit der paradoxen Konstellation, daß Becketts Figuren ihr Glück in der Hölle suchen. Obwohl sie auf ewig verdammt sind, gelingt es ihnen, sich durch Reden und Erfinden der Illusion der Freiheit hinzugeben - als befänden sie sich außerhalb der Welt und wären weder lebendig noch tot. Sie leben in einer unmöglich, eben absurd anmutenden Unsterblichkeit und können sich auf diese Weise dem Fiasko der Realität, der "condition humaine", entziehen. Eine inflationäre Verwendung des Begriffs der Absurdität seit den Anfängen der Beckett-Forschung hat allerdings seine Aussagekraft erheblich reduziert: Von dieser Feststellung ausgehend schlägt Carola Veit vor, auf die statisch existenzialistische Bedeutung - synonym mit Sinnlosigkeit, Hoffnungslosigkeit - zu verzichten. Denn Becketts Figuren empfinden ihre Situation nicht als absurd; sie erscheinen absurd, weil der Leser oder Zuschauer in ihnen einen logischen Sinn sucht. Becketts Schreibweise jedoch, die systematisch dekonstruiert, verwehrt dem Leser den rationalen Nachvollzug und damit die dualistische Fixierung der Unbegreiflichkeit. Wie Federman wendet sich auch Martin Brunkhorst dagegen, daß die ernsthaft existenzialistische Interpretationsweise die Komik der Texte verdrängt hat. Mit einem Vergleich zwischen Grabbes Lustspiel Scherz, Satire, Ironie und tiefere Bedeutung, einem bekannten Vorläufer des modernen Theaters, und Krapp's Last Tape verdeutlicht er, daß der tiefere Sinn der Beckettschen Texte sich erst vor einem tragischen Hintergrund entfaltet. Er plädiert außerdem dafür, daß man die clownesken Elemente, die leisen ironischen Töne und Gesten dieses Kopf-Theaters durch eine entsprechende Aufführungspraxis wieder freilegen sollte. Manfred Schmeling analysiert das Konzept des Beckettschen Humors: Ihm liegt der Dualismus von Körper und Geist zugrunde. Diese philosophische Idee wird allerdings kraft des literarischen Spiels ins Ironische und Burleske gewen- 
det. Daraus entspringt ein Lachen ohne Versöhnung, weil die ethischen Parameter, die etwa die Komiktheorie Bachtins enthält, außer Kraft gesetzt werden. Durch den ironischen Blick der Erzähler auf die Körperlichkeit - worauf Schmeling mit dem Motiv der "Fenstervariante" hinweist - wird die monadische Einsamkeit des Geistes hintergangen. Auch Wilhelm Füger stellt fest, daß die sogenannten natürlichen Funktionen des Lachens von Becketts Werk nicht mehr ausgelöst werden. Das Lachen der Protagonisten richtet sich gegen sie selbst; es ist ein Lachen ohne Folgen. Beckett nannte es den "risus purus": "das über das Lachen lachende Lachen" (Watt). Diese Vorstellung ergibt sich Füger zufolge daraus, daß Beckett sich mit Mauthners Sprachkritik beschäftigt hat. Dieser verstand Lachen und Schweigen als Protest gegen die Unzulänglichkeit der Sprache. Becketts Bestreben war es daher, eine Sprache zu finden, die sich dem Schweigen annähert und noch Raum für das Lachen läßt. Konrad Schoell untersucht die verschiedenen Arten des Lachens der Figuren Becketts; er bezieht die Wirkung auf den Zuschauer mit ein. Neben den bekannteren Formen des Komischen finden sich in Becketts Texten vor allem logische und kombinatorische Spiele, Collagen oder Juxtapositionen, mit denen weit auseinanderliegende inkongruente Bereiche nebeneinandergesetzt werden. Der "risus purus" als Lachen über das eigenen Unglück läßt sich jedoch nicht unvermittelt auf den Zuschauer übertragen, weil dieser nicht in entsprechendem Maße abgeklärt und gelassen ist. Chris Rauseo führt die Komik bei Beckett auf die rhetorische Kategorie der Angemessenheit zurück. Er arbeitet die stilistische Diskrepanz zwischen Form und Inhalt heraus: Irische Witze werden auf französisch erzählt. Sprachliche Convenance und Bienséance des Französischen kollidieren mit den niedrigen Inhalten der Texte, den Schlammszenarien. Insofern können Becketts französische Texte komischer als seine englischen wirken.

Auch Robert Kudielka widmet sich den produktionsästhetischen Aspekten unserer Fragestellung. Er untersucht Becketts Auseinanderset- 
zung mit der modernen Kunst. In Malerei und Dichtung manifestiert sich eine Krise der Beziehung zwischen der Darstellung und ihrem Anlaß. Die Ungewißheit dieser Beziehung äußert sich 'positiv' in einer selbständigeren Rolle der darstellerischen Mittel. Beckett ist der "Reinheit der Mittel" am nächsten gekommen. Die Semantik des sprachlichen Ausdrucks bringt allerdings eine komödiantische Note ins Spiel, welche der Kunst fremd ist. An die Stelle des existentiellen Verhängnisses tritt die unauflösbare Komödie. Therese Fischer-Seidel begründet die abstrakte visuelle Wahrnehmung, wie sie sich in Becketts Werken niedergeschlagen hat, mit der Gestaltpsychologie, die er kannte. Im Frühwerk werden Erkenntnisse der Wahrnehmungs- und Gestaltpsychologie beschreibend aufgenommen; im späten dramatischen Werk werden sie nach dem Prinzip der Kippbilder literarisch realisiert. Dieses Verfahren der Verdopplung begründet Marianne Kesting anhand der Erzählerfiguren psychoanalytisch. Die Wahrnehmung des Künstlers führt über die Introspektion, die Abwendung von der äußeren Welt in die Imagination, die den Solipsismus voraussetzt: Die Figuren entstehen aus Visionen bei geschlossenen Lidern. Die Abspaltungen des Ichs formieren sich zu Phantasiefiguren; diese Figuren bewegen sich im Raum ihres Geistes. Ähnlich erlebt sich auch der Leser als erfindender und interpretierender. Jean Bessière widmet sich späten Texten, deren Titel man als Indikatoren der Ich-Auflösung wie der ironischen IchBehauptung verstehen kann. Nach Bessière manifestiert sich der Solipsismus als Sicht von außen auf einen in sich gefangenen Geist. Die Ironie resultiert aus den besonderen Inhalten der Rede des mit sich selbst sprechenden Solipsisten. Erzählen ist Setzen und Aufheben in der Zeit. Solipsismus und Ironie erscheinen als literarische Verfahren, um die individuelle Rede zugunsten einer abstrakten Rede aufzuheben; dieses können wir als einen Versuch verstehen, die Ungewißheit der Beziehung zwischen der Darstellung und ihrem Anlaß (R. Kudielka) literarisch zu verwirklichen. In den späten Texten kommt es Beckett offensichtlich immer weniger auf das Dargestellte, "ces contes-comptes", als auf den Aus- 
druck des Darstellens selbst an, "le processus". Peter Brockmeier geht von der Vervielfältigung der Erzähler oder Erzählfiguren aus, die einer im Selbstgespräch versunkenen Erzähler-Instanz entspringen. Indem er auf Arthur Koestlers Theorie des Komischen als Teil seiner Theorie der Kreativität zurückgreift, sucht er in der scheinbar absoluten Freiheit des mit sich sprechenden Erzählers, also in seinem Spiel mit sich selbst, den Ursprung der komischen Wirkung der Texte Becketts.

Wir danken unseren Autorinnen und Autoren, die unter extremen klimatischen Bedingungen an dem Kolloquium mitgewirkt und ihre Manuskripte unter empfindlichem Zeitdruck für den Druck vorbereitet haben.

Die Durchführung des Kolloquiums wurde durch die großzügige finanzielle Unterstützung der Forschungsabteilung und der Philosophischen Fakultät II der Humboldt-Universität ermöglicht; dem Institut Français de Berlin danken wir dafür, daß es die Einladung eines französischen Kollegen übernommen hat. Die Veröffentlichung konnte dank eines Druckkostenzuschusses der Philosophischen Fakultät II verwirklicht werden.

Wir bedanken uns bei dem Verlag, daß er den Band kurzfristig in sein Programm aufgenommen hat.

Berlin, im August 1996.

Peter Brockmeier Carola Veit 